56, 3, pp. 585-599, Warsaw 2018

DOI: $10.15632 /$ jtam-pl.56.3.585

\title{
BIFURCATION AND CHAOS ANALYSIS OF A GEAR-ROTOR-BEARING SYSTEM
}

\author{
Xiangfeng Gou \\ School of Mechanical Engineering, Tianjin Polytechnic University, Tianjin, China \\ Tianjin Key Laboratory of Advanced Mechatronics Equipment Technology, Tianjin, China \\ School of Mechanical Engineering, Lanzhou Jiaotong University, Lanzhou, China \\ e-mail: 20150022@tjpu.edu.cn
}

LINGYUN ZHU

School of Mechanical Engineering, Tianjin Polytechnic University, Tianjin, China

School of Mechanical Engineering, Lanzhou Jiaotong University, Lanzhou, China

Changuun QI

School of Mechanical Engineering, Lanzhou Jiaotong University, Lanzhou, China

\begin{abstract}
To study chaos and bifurcation of a gear system, a five-degree-of-freedom nonlinear dynamic model of a gear-rotor-bearing system is established. It consists of a gear pair, supporting shafts, bearings and other auxiliary components. The effects of frequency, backlash, bearing clearance, comprehensive transmission error and stiffness on nonlinear dynamics of the system are investigated according to bifurcation diagrams, phase portraits and Poincaré maps by a numerical method. Some nonlinear phenomena such as grazing bifurcation, Hopf bifurcation, inverse-Hopf bifurcation, chaos and coexistence of attractors are investigated. Different grazing bifurcations and their causes are discussed. The critical parameters are identified, too.
\end{abstract}

Keywords: gear-rotor-bearing, dynamics, bifurcation, chaos

\section{Introduction}

Gear systems play a major role in mechanical engineering and other engineering fields. There are plenty of researches on nonlinear dynamics of gear systems since Kahraman and Singh (1990) researched nonlinear dynamics of a spur gear pair where the backlash was represented by a truncated series expansion. They developed a 3-DOF dynamic model including non-linearities associated with radial clearances in the radial rolling element bearings and backlash between a spur gear pair in 1991. Ranghothama and Narayanan (1999) investigated periodic motions and chaotic motions of a nonlinear geared rotor-bearing system. Theodossiades and Natsiavas (2001) investigated the response and stability characteristics of a gear pair system supported on oil journal bearings. Choi et al. (2001) analyzed dynamic characteristics of a geared rotor-bearing system with the transfer matrix method. Chen et al. (2011) developed a multi-degree-of-freedom nonlinear dynamic gear system with dynamic backlash, friction and time varying stiffness. Chang-Jian (2010a,b) established a dynamical model of a HSFD mounted gear-bearing system, a dynamical model of a gear-bearing system under nonlinear suspension, Chang-Jian and Chang (2012a,b) - a dynamical model of the porous squeeze film damper mounted on a gear-bearing system and a dynamical model of a gear pair system equipped with journal bearings under turbulent low. Cui et al. (2012) established a dynamical model of a geared rotor system with a nonlinear oil film force and a nonlinear mesh force. Xiang et al. (2016) proposed a period 
expansion method to build a 6-DOF nonlinear dynamic model of a spur gear pair with time varying stiffness, gear backlash and surface friction.

The motor and other auxiliary components mounted on the input and output shafts as rotor are abstracted as rotors coupled with the gear-bearing system in this paper. A 5-DOF nonlinear dynamic model which is closer to the engineering practice is developed to research the bifurcation and chaos of the system. It is a multiple clearance and multi-parameter coupled system.

\section{Nonlinear dynamic model of a gear-rotor-bearing system}

A simplified theoretical model of a gear-rotor-bearing system is illustrated in Fig. 1. The gear meshing part is simplified as two rotors coupled with viscous dampers and nonlinear springs. Three nonlinear factors such as time-varying stiffness, backlash and the comprehensive transmis-

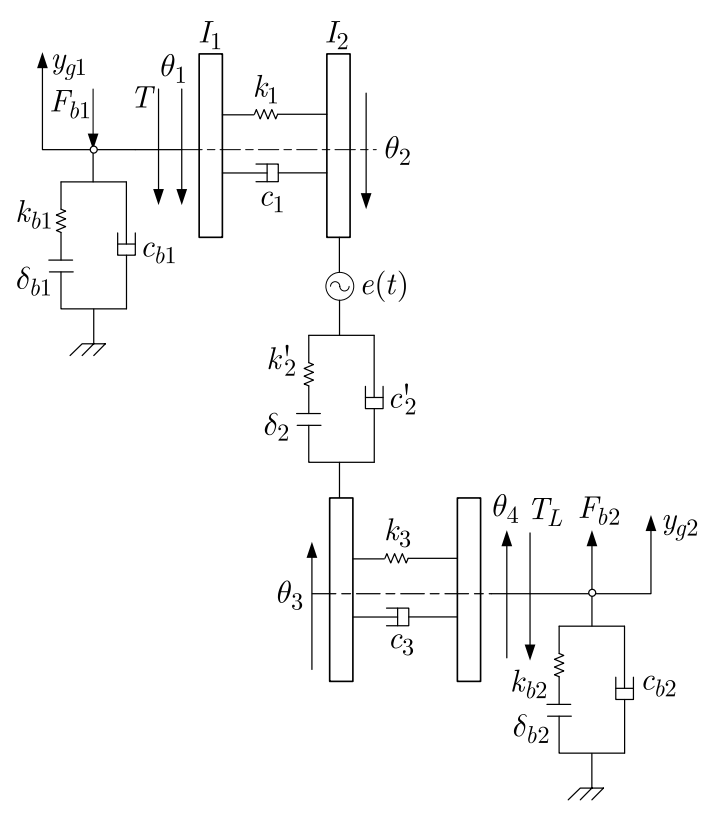

Fig. 1. Dynamic model of the gear-rotor-bearing system

sion error are considered in it. Axial vibration, transverse vibration and longitudinal vibration are not taken into account. Torsional vibration displacements between meshing gears are studied only. The torsional vibration displacement of the two gears and rotors is denoted as $\theta_{i}$ $(i=1,2,3,4)$, respectively. The mass moment of inertia of the two gears is $I_{i}(i=2,3)$ and the moment of inertia of the two rotors is $I_{i}(i=1,4)$, respectively. The base circle radius of the two gears is $r_{b i}(i=2,3)$, respectively. Torsional damping is $c_{1}$ and $c_{3}$ and the torsional stiffness of the two rotation shafts is $k_{1}$ and $k_{3}$, respectively. The mesh stiffness and damping coefficient is $k_{2}^{\prime}$ and $c_{2}^{\prime}$, respectively. The force of gears by bearings on the input and output shafts is $F_{b i}$ $(i=1,2)$, respectively. The torque of the input and output shafts is $T$ and $T_{L}$, respectively. The damping coefficient of the bearings on the input and output shafts is $c_{b i}(i=1,2)$, respectively. The average support stiffness of the bearings on the input and output shafts is $k_{b i}(i=1,2)$, respectively. The backlash function $f(\cdot)$ is usually used to represent gear clearance. The bearing clearance functions on the input and output shafts is $\bar{f}(\cdot)(i=1,2)$, respectively. The actual backlash is $\delta_{2}$. The bearing clearance on the input and output shafts is $\delta_{b i}(i=1,2)$, respectively. The displacement of the center of the two gears is $y_{g i}(i=1,2)$, respectively. The time-varying comprehensive transmission error of the gear pair is $e(t)$. The absolute rotational equations of the system can be derived according to the 2nd Newton's law 


$$
\begin{aligned}
& I_{1} \ddot{\theta}_{1}+c_{1}\left(\dot{\theta}_{1}-\dot{\theta}_{2}\right)+k_{1}\left(\theta_{1}-\theta_{2}\right)=T \\
& I_{2} \ddot{\theta}_{2}-c_{1}\left(\dot{\theta}_{1}-\dot{\theta}_{2}\right)-k_{1}\left(\theta_{1}-\theta_{2}\right)+R_{b 2} c_{2}^{\prime}\left[R_{b 2} \dot{\theta}_{2}-R_{b 3} \dot{\theta}_{3}-\dot{e}(t)-\left(\dot{y}_{g 1}-\dot{y}_{g 2}\right)\right] \\
& \quad+R_{b 2} k_{2}^{\prime} f\left[R_{b 2} \theta_{2}-R_{b 3} \theta_{3}-e(t)-\left(y_{g 1}-y_{g 2}\right)\right]=0 \\
& \quad I_{3} \ddot{\theta}_{3}-c_{3}\left(\dot{\theta}_{3}-\dot{\theta}_{4}\right)+k_{1}\left(\theta_{1}-\theta_{2}\right)-R_{b 3} c_{2}^{\prime}\left[R_{b 2} \dot{\theta}_{2}-R_{b 3} \dot{\theta}_{3}-\dot{e}(t)-\left(\dot{y}_{g 1}-\dot{y}_{g 2}\right)\right] \\
& \quad-R_{b 3} k_{2}^{\prime} f\left[R_{b 2} \theta_{2}-R_{b 3} \theta_{3}-e(t)-\left(y_{g 1}-y_{g 2}\right)\right]=0 \\
& I_{4} \ddot{\theta}_{4}+c_{3}\left(\dot{\theta}_{3}-\dot{\theta}_{4}\right)+k_{3}\left(\theta_{3}-\theta_{4}\right)=-T_{L}
\end{aligned}
$$

The theoretical backlash is defined as $2 b$, and $b$ is selected as the feature size. The relative rotational equation of the system can be written as in Eqs. (2.2) by subtracting every two in Eqs. (2.1) with the following expressions $\gamma=b / R_{b 2}, q_{1}=\left(\theta_{1}-\theta_{2}\right) / \gamma, q_{2}=\left[R_{b 2} \theta_{2}-R_{b 3} \theta_{3}-\right.$ $\left.e(t)-\left(y_{g 1}-y_{g 2}\right)\right] / b$ and $q_{3}=\left(\theta_{3}-\theta_{4}\right) / \gamma$

$$
\begin{aligned}
\ddot{q}_{1} & +\left(\frac{c_{1}}{I_{1}}+\frac{c_{1}}{I_{2}}\right) \dot{q}_{1}+\left(\frac{k_{1}}{I_{1}}+\frac{k_{1}}{I_{2}}\right) q_{1}-\frac{R_{b 2}^{2} c_{2}^{\prime}}{I_{2}} \dot{q}_{2}-\frac{R_{b 2}^{2} k_{2}^{\prime}}{I_{2}} f\left(q_{2}\right)=\frac{T}{I_{1} \gamma} \\
\ddot{q}_{2}- & \frac{c_{1}}{I_{2}} \dot{q}_{1}-\frac{k_{1}}{I_{2}} q_{1}-\frac{R_{b 3} c_{3}}{I_{3} R_{b 2}} \dot{q}_{3}-\frac{R_{b 3} k_{3}}{I_{3} R_{b 2}} q_{3}+\left(\frac{R_{b 2}^{2} c_{2}^{\prime}}{I_{2}}+\frac{R_{b 3}^{2} c_{2}^{\prime}}{I_{3}}\right) \dot{q}_{2} \\
& +\left(\frac{R_{b 2}^{2} k_{2}^{\prime}}{I_{2}}+\frac{R_{b 3}^{2} k_{2}^{\prime}}{I_{3}}\right) f\left(q_{2}\right)=\frac{-\ddot{e}(t)-\left(\ddot{y}_{g 1}-\ddot{y}_{g 2}\right)}{b} \\
\ddot{q}_{3} & +\left(\frac{c_{3}}{I_{3}}+\frac{c_{3}}{I_{4}}\right) \dot{q}_{3}+\left(\frac{k_{3}}{I_{3}}+\frac{k_{3}}{I_{4}}\right) q_{3}-\frac{R_{b 2} R_{b 3} c_{2}^{\prime}}{I_{3}} \dot{q}_{2}-\frac{R_{b 2} R_{b 3} k_{2}^{\prime}}{I_{3}} f\left(q_{2}\right)=\frac{T_{L}}{I_{4} \gamma}
\end{aligned}
$$

The dimensionless torsional vibration equation of the system can be obtained by defining $\tau=\omega_{n} t, \dot{q}_{(1,2,3)}=\dot{q}_{(1,2,3)} / \omega_{n}^{2}, \dot{y}_{(g 1, g 2)}=b \omega_{n} \dot{q}_{(4,5)}$ where $\omega_{n}=\sqrt{k_{2}^{\prime} I_{2} I_{3} /\left(I_{3} R_{b 2}^{2}+I_{2} R_{b 3}^{2}\right)}$

$$
\begin{aligned}
& \ddot{q}_{1}+\xi_{11} \dot{q}_{1}-\xi_{12} \dot{q}_{2}+k_{11} q_{q}-k_{12}[1+k \cos (\tau+\varphi)] f\left(q_{2}\right)=F_{m i}+F_{a i} \sin \left(\tau+\varphi_{i}\right) \\
& \ddot{q}_{2}-\xi_{21} \dot{q}_{1}+\xi_{22} \dot{q}_{2}-\xi_{23} \dot{q}_{3}-k_{21} q_{1}+k_{22}[1+k \cos (\tau+\varphi)] f\left(q_{2}\right)-k_{23} q_{3} \\
& \quad=-\omega^{2} \varepsilon \sin (\tau+\varphi)-\ddot{q}_{4}+\ddot{q}_{5} \\
& \ddot{q}_{3}-\xi_{31} \dot{q}_{2}+\xi_{32} \dot{q}_{3}-k_{31}[1+k \cos (\tau+\varphi)] f\left(q_{2}\right)+k_{32} q_{3}=F_{m l}+F_{a l} \sin \left(\tau+\varphi_{o}\right)
\end{aligned}
$$

The dimensionless parameters are defined as

$$
\begin{aligned}
& \xi_{11}=\frac{c_{2}^{\prime} R_{b 2}^{2} R_{b 3}}{I_{1} I_{2} \omega_{n}} \quad \xi_{12}=\frac{R_{b 2}^{2} c_{2}^{\prime}}{I_{2} \omega_{n}} \quad \xi_{23}=\frac{c_{3} R_{b 3}}{I_{3} R_{b 2} \omega_{n}} \\
& \xi_{21}=\frac{c_{1}}{I_{2} \omega_{n}} \quad \xi_{22}=\frac{c_{2}^{\prime}\left(I_{3} R_{b 2}^{2}+I_{2} R_{b 3}^{2}\right)}{I_{2} I_{3} \omega_{n}} \quad \xi_{31}=\frac{c_{2}^{\prime} R_{b 2}^{2} R_{b 3}}{I_{3} R_{b 2} \omega_{n}} \\
& \xi_{32}=\frac{c_{3}\left(I_{3}+I_{4}\right)}{I_{3} I_{4} \omega_{n}} \quad k_{11}=\frac{k_{1}\left(I_{1}+I_{2}\right)}{I_{1} I_{2} \omega_{n}^{2}} \quad k_{12}=\frac{R_{b 2}^{2} k_{2}^{\prime}}{I_{2} \omega_{n}^{2}} \\
& k_{21}=\frac{k_{1}}{I_{2} \omega_{n}^{2}} \quad k_{22}=\frac{k_{2}^{\prime}\left(I_{3} R_{b 2}^{2}+I_{2} R_{b 3}^{2}\right)}{I_{2} I_{3} \omega_{n}^{2}} \quad k_{23}=\frac{k_{3} R_{b 3}}{I_{3} R_{b 2} \omega_{n}^{2}} \\
& k_{31}=\frac{k_{2}^{\prime} R_{b 2}^{2} R_{b 3}}{I_{3} R_{b 2} \omega_{n}^{2}} \quad k_{32}=\frac{k_{3}\left(I_{3}+I_{4}\right)}{I_{3} I_{4} \omega_{n}}
\end{aligned}
$$

in equations (2.3). The time-varying mesh stiffness is $k(t)=1+k \sin (\omega t+\varphi)$ (Kahraman and Singh, 1990) where the dimensionless mesh frequency is $\omega=\omega_{e} / \omega_{n}$ and $\omega_{e}$ is the mesh frequency of the gear. The dynamical transmission error is $e(t)=1+\varepsilon \sin (\omega t+\varphi)$ (Kahraman and Singh, 1990). The dimensionless loads can be defined as

$$
F_{m i}=\frac{T}{I_{1} \gamma} \quad F_{a i}=\frac{T \sin \left(\omega t+\varphi_{i}\right)}{I_{1} \gamma} \quad F_{m l}=\frac{T_{L}}{I_{4} \gamma} \quad F_{a l}=\frac{T_{L} \sin \left(\omega t+\varphi_{o}\right)}{I_{4} \gamma}
$$

where $\varphi_{i}$ and $\varphi_{o}$ is the initial phase angular of the excitation and response, respectively. 
Transverse vibration equations of the system can be formulated according to the 2nd Newton's law

$$
\begin{aligned}
& m_{g 1} \ddot{y}_{g 1}+c_{b 1} \dot{y}_{g 1}-c_{2}^{\prime}\left[R_{b 2} \dot{\theta}_{2}-R_{b 3} \dot{\theta}_{3}-\dot{e}(t)-\left(\dot{y}_{g 1}-\dot{y}_{g 2}\right)\right]+k_{b 1} \bar{f}_{b 1}\left(y_{g 1}\right) \\
& \quad-k_{2}^{\prime} f\left[R_{b 2} \theta_{2}-R_{b 3} \theta_{3}-e(t)-\left(y_{g 1}-y_{g 2}\right)\right]=-F_{b 1} \\
& m_{g 2} \ddot{y}_{g 2}+c_{b 2} \dot{y}_{g 2}+c_{2}^{\prime}\left[R_{b 2} \dot{\theta}_{2}-R_{b 3} \dot{\theta}_{3}-\dot{e}(t)-\left(\dot{y}_{g 1}-\dot{y}_{g 2}\right)\right]+k_{b 2} \bar{f}_{b 2}\left(y_{g 2}\right) \\
& \quad+k_{2}^{\prime} f\left[R_{b 2} \theta_{2}-R_{b 3} \theta_{3}-e(t)-\left(y_{g 1}-y_{g 2}\right)\right]=F_{b 2}
\end{aligned}
$$

where the mass of the gear and rotor on the drive and driven shaft is $m_{g 1}$ and $m_{g 2}$, respectively.

The dimensionless transverse vibration equations of the system can be obtained when the dimensionless parameters are defined as

$$
\begin{array}{llll}
\xi_{41}=\frac{c_{b 1}}{2 m_{g 1} \omega_{n}} & \xi_{42}=\frac{c_{2}^{\prime}}{2 m_{g 1} \omega_{n}} & \xi_{51}=\frac{c_{b 2}}{2 m_{g 2} \omega_{n}} & \xi_{52}=\frac{c_{2}^{\prime}}{2 m_{g 2} \omega_{n}} \\
k_{41}=\frac{k_{b 1}}{m_{g 1} \omega_{n}^{2}} & k_{42}=\frac{k_{2}^{\prime}}{m_{g 2} \omega_{n}^{2}} & k_{51}=\frac{k_{b 2}}{m_{g 2} \omega_{n}^{2}} & k_{52}=\frac{k_{2}^{\prime}}{m_{g 2} \omega_{n}^{2}} \\
\bar{F}_{b 1}=\frac{F_{b 1}}{m_{g 1} b \omega_{n}^{2}} & \bar{F}_{b 2}=\frac{F_{b 2}}{m_{g 2} b \omega_{n}^{2}} & &
\end{array}
$$

and

$$
\begin{aligned}
& \ddot{q}_{4}+2 \xi_{41} \dot{q}_{4}+k_{41} \bar{f}_{b 1}\left(q_{4}\right)-2 \xi_{42} \dot{q}_{2}-k_{42} f\left(q_{2}\right)=-\bar{F}_{b 1} \\
& \ddot{q}_{5}+2 \xi_{51} \dot{q}_{5}+k_{51} \bar{f}_{b 2}\left(q_{5}\right)+2 \xi_{52} \dot{q}_{2}+k_{52} f\left(q_{2}\right)=\bar{F}_{b 2}
\end{aligned}
$$

A 5-DOF dimensionless dynamical equation of the gear-rotor-bearing system can be obtained by coupling the torsional vibration and transverse vibration. It can be written as the state equation by defining $x_{1}=q_{1}, x_{2}=\dot{x}_{1}, x_{3}=q_{2}, x_{4}=\dot{x}_{3}, x_{5}=q_{3}, x_{6}=\dot{x}_{5}, x_{7}=q_{4}, x_{8}=\dot{x}_{7}$, $x_{9}=q_{5}, x_{10}=\dot{x}_{9}$

$$
\begin{aligned}
& \dot{x}_{1}=x_{2} \\
& \dot{x}_{2}=F_{m i}+F_{a i} \sin \left(\tau+\varphi_{i}\right)-\xi_{11} x_{2}+\xi_{12} x_{4}-k_{11} x_{1}+k_{12}[1+k \cos (\tau+\varphi)] f\left(x_{3}\right) \\
& \dot{x}_{3}=x_{4} \\
& \dot{x}_{4}=-\dot{x}_{8}+\dot{x}_{10}+\xi_{21} x_{2}-\xi_{22} x_{4}+\xi_{23} x_{6}+k_{21} x_{1}-k_{22}[1+k \cos (\tau+\varphi)] f\left(x_{3}\right) \\
& \quad+k_{23} x_{5}-\omega^{2} \varepsilon \sin (\tau+\varphi) \\
& \dot{x}_{5}=x_{6} \\
& \dot{x}_{6}=F_{m l}+F_{a l} \sin \left(\tau+\varphi_{o}\right)+\xi_{31} x_{4}-\xi_{32} x_{6}+k_{31}[1+k \cos (\tau+\varphi)] f\left(x_{3}\right)-k_{33} x_{5} \\
& \dot{x}_{7}=x_{8} \\
& \dot{x}_{8}=-\bar{F}_{b 1}-2 \xi_{41} x_{8}-k_{41} f_{b 1}\left(x_{7}\right)+2 \xi_{42} x_{4}+k_{42} f\left(x_{3}\right) \\
& \dot{x}_{9}=x_{10} \\
& \dot{x}_{10}=\bar{F}_{b 2}-2 \xi_{51} x_{10}-k_{51} f_{b 2}\left(x_{9}\right)-2 \xi_{52} x_{4}-k_{52} f\left(x_{3}\right)
\end{aligned}
$$

Backlash is one of the main nonlinear factors in the gear system. The backlash function of the gear pair can be written as follows if the dimensionless backlash is defined as $D=\delta_{2} /(2 b)$

$$
f(x)= \begin{cases}x-D & \text { for } \quad x>D \\ 0 & \text { for } \quad-D \leqslant x \leqslant D \\ x+D & \text { for } \quad x<-D\end{cases}
$$

The rolling bearing is composed of the inner ring, outer ring and rolling elements. Clearance exists between the inner ring and rolling elements or rolling elements and the outer ring. It is 
one of the main nonlinear factors in the system, too. The bearing clearance functions can be drawn up as follows if the dimensionless bearing clearance is defined as $D_{b i}=\delta_{b i} /(2 b)(i=1,2)$

$$
\begin{aligned}
& \bar{f}_{b 1}\left(x_{7}\right)=\left\{\begin{array}{lll}
x_{7}-D_{b 1} & \text { for } & x_{7}>D_{b 1} \\
0 & \text { for } & -D_{b 1} \leqslant x_{7} \leqslant D_{b 1} \\
x_{7}+D_{b 1} & \text { for } & x_{7}<-D_{b 1}
\end{array}\right. \\
& \bar{f}_{b 2}\left(x_{9}\right)=\left\{\begin{array}{lll}
x_{9}-D_{b 2} & \text { for } & x_{9}>D_{b 2} \\
0 & \text { for } & -D_{b 2} \leqslant x_{9} \leqslant D_{b 2} \\
x_{9}+D_{b 2} & \text { for } & x_{9}<-D_{b 2}
\end{array}\right.
\end{aligned}
$$

\section{Bifurcation and chaos of the system}

A gearbox of a metro train is selected as the research object. The material of the gear is $40 \mathrm{Cr}$. The related parameters of the gearbox are given in Table 1 . The lengths of two shafts are $450 \mathrm{~mm}$. The elastic modulus of the shafts is $2 \cdot 10^{11} \mathrm{~Pa}$, the Poisson ratio is 0.3 . Each shaft is supported by two bearings, 7306 . The preload load of the bearing is $200 \mathrm{~N}$. The torque is $150 \mathrm{~N} \cdot \mathrm{m}$. The related parameters of bearings are given in Table 2 . The calculated dimensionless parameters are given in Table 3. Other dimensionless coefficients of the time-varying stiffness can be calculated by computer programs according to the meshing parameters real-timely.

Table 1. Parameters of the baseline example gear pair

\begin{tabular}{|l|c|c|l|c|c|}
\hline System parameters & Pinion & Gear & \multicolumn{1}{|c|}{ System parameters } & Pinion & Gear \\
\hline \hline Number of teeth $z$ & 40 & 156 & Radius of base circle $r_{b}[\mathrm{~mm}]$ & 56.4 & 219.9 \\
\hline Mass $[\mathrm{kg}]$ & 1.82 & 2.63 & $\begin{array}{l}\text { Mass moment of inertia } \\
\text { of gear }\left[\times 10^{-3} \mathrm{~kg} \cdot \mathrm{m}^{2}\right]\end{array}$ & 2.6 & 3.1 \\
\hline Module $[\mathrm{mm}]$ & 3 & 3 & $\begin{array}{l}\text { Mass moment of inertia } \\
\text { of rotor }\left[\times 10^{-3} \mathrm{~kg} \cdot \mathrm{m}^{2}\right]\end{array}$ & 3.4 & 2.8 \\
\hline Backlash $[\mu \mathrm{m}]$ & 100 & 100 & Meshing damping ratio & 0.1 & 0.1 \\
\hline Width of gear $[\mathrm{mm}]$ & 52 & 48 & Pressure angle $\left.\alpha{ }^{\circ}\right]$ & 20 & 20 \\
\hline
\end{tabular}

Table 2. Parameters of bearings

\begin{tabular}{|l|c|l|c|}
\hline \multicolumn{1}{|c|}{ System parameters } & Value & \multicolumn{1}{c|}{ System parameters } & Value \\
\hline \hline Inner radius $[\mathrm{mm}]$ & 30 & Race way curvity of inner ring & 0.52 \\
\hline Outer radius $[\mathrm{mm}]$ & 62 & Race way curvity of outer ring & 0.525 \\
\hline Radius of rolling $[\mathrm{mm}]$ & 9.525 & Radial clearance $[\mu \mathrm{m}]$ & 60 \\
\hline Number of rolling elements & 11 & & \\
\hline
\end{tabular}

Table 3. Value of dimensionless parameters

\begin{tabular}{|c|c|c|c|c|c|c|c|c|c|}
\hline Param. & Value & Param. & Value & Param. & Value & Param. & Value & Param. & Value \\
\hline \hline$k_{11}$ & 1.05 & $k_{33}$ & 1.05 & $\xi_{11}$ & 0.1 & $\xi_{32}$ & 0.1 & $k_{52}$ & 0.6 \\
\hline$k_{12}$ & 0.6 & $k_{31}$ & 0.3 & $\xi_{12}$ & 0.1 & $\xi_{31}$ & 0.1 & $\xi_{52}$ & 0.125 \\
\hline$k_{21}$ & 0.6 & $k_{41}$ & 1.1 & $\xi_{22}$ & 0.2 & $\xi_{41}$ & 0.01 & $\bar{F}_{b 2}$ & 0.1 \\
\hline$k_{23}$ & 0.7 & $k_{42}$ & 0.6 & $\xi_{21}$ & 0.05 & $\xi_{42}$ & 0.125 & $F_{a i}$ & 0.1 \\
\hline$k_{22}$ & 0.8 & $k_{51}$ & 1.1 & $\xi_{23}$ & 0.05 & $\xi_{51}$ & 0.01 & $F_{m l}$ & 0.1 \\
\hline$F_{m i}$ & 0.05 & $\bar{F}_{b 1}$ & 0.1 & $F_{a l}$ & 0.2 & & & & \\
\hline
\end{tabular}




\subsection{Effect of the mesh frequency}

The bifurcation diagram of the relative displacement via the frequency, $\omega \in[0.5,2.5]$ as $D=1.0$ and $D_{b}=D_{b 1}=D_{b 2}=0.6$ is illustrated in Fig. 2. The bifurcation and chaos of the system with the increasing frequency will be discussed in three regions precisely.

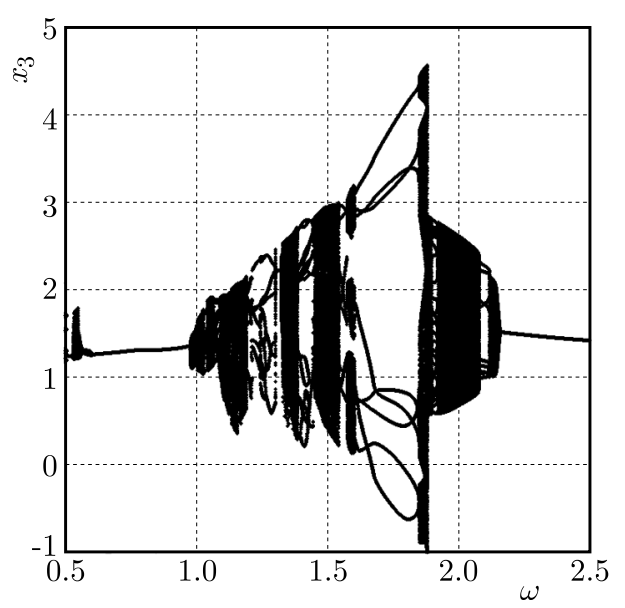

Fig. 2. Bifurcation diagram of the system via the mesh frequency

(a)

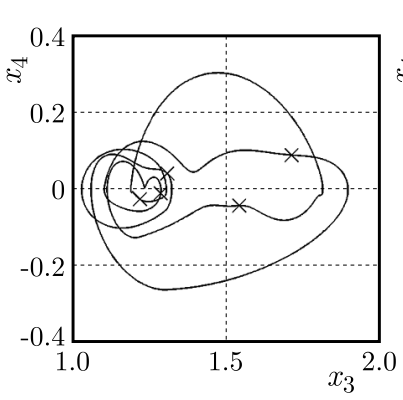

(b)

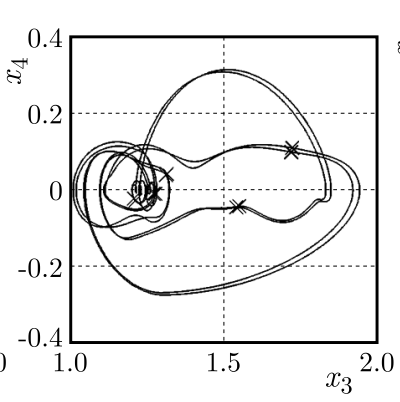

(c)

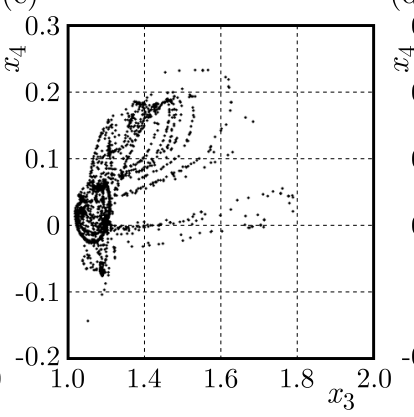

(d)

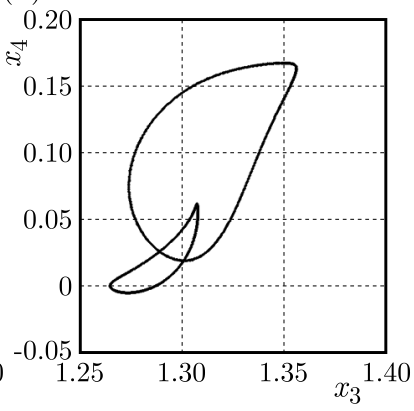

Fig. 3. Diagrams of Poincaré mapping and phase portraits: (a) $\omega=0.5$, (b) $\omega=0.502$, (c) $\omega=0.545$, (d) $\omega=0.56$. Here, the diagram of Poincaré mapping in $a$ and $b$ is represented by $\times$

Firstly, the bifurcation and chaos of the system as $\omega \in[0.5,0.62]$ is studied. The motion of the system is stable period-5 motion as $\omega=0.5$. The diagram of Poincaré mapping and phase portrait is illustrated in Fig. 3a as $\omega=0.5$ and the diagram of Poincaré mapping is represented by $\times$. There are 5 discrete points in the diagram of Poincaré mapping. Doubling periodic bifurcation occurs and the period-5 motion doubles to period-10 motion when the frequency $\omega$ increases to $\omega=0.501$. The diagram of Poincaré mapping and phase portrait is illustrated in Fig. 3b as $\omega=0.502$. The period-10 degenerates to period- 5 when the frequency $\omega$ increases to 0.503 . The period- 5 motion transits to period- 1 motion when $\omega$ increases to 0.505 . Doubling periodic bifurcation occurs with an increase in the mesh frequency and the period-1 motion doubles to stable period- 2 motion as $\omega=0.5125$. Saddle-node bifurcation leads the system to chaotic motion as $\omega=0.5315$. The diagram of Poincaré mapping is illustrated in Fig. 3c as $\omega=0.545$. The chaotic motion of the system exists in a small range and it evolves in quasi-periodic motion by Hopf bifurcation when $\omega$ increases to 0.556. A diagram of Poincaré mapping is presented in Fig. 3d. There are two closed tori in the diagram of Poincaré mapping. Inverse Hopf bifurcation occurs when $\omega$ increases to 0.565 and it leads the quasi-periodic motion to period-2 motion. Inverse-doubling periodic bifurcation leads the period- 2 motion to period-1 motion when $\omega$ increases in 0.602 . The period-1 motion exists in a considerable range of frequency. 
(a)
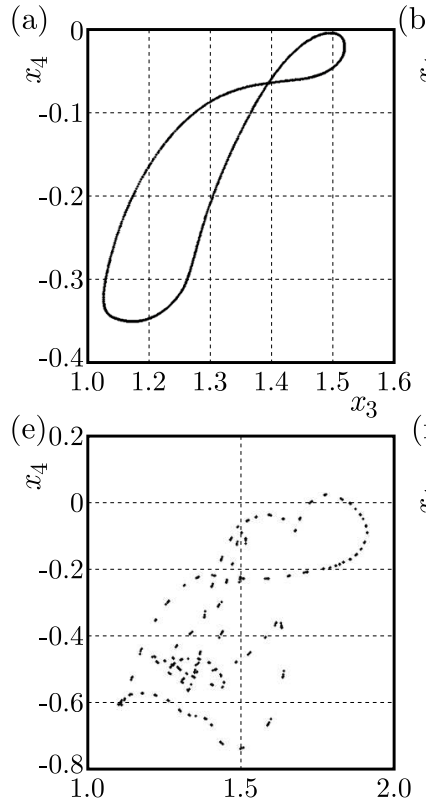

(i)

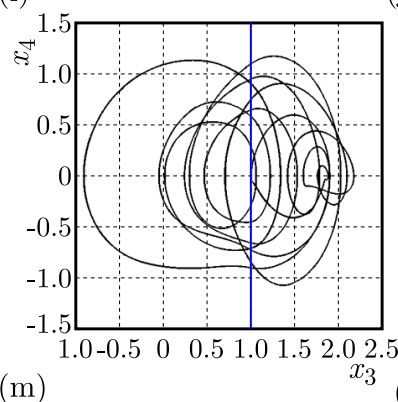

(m)
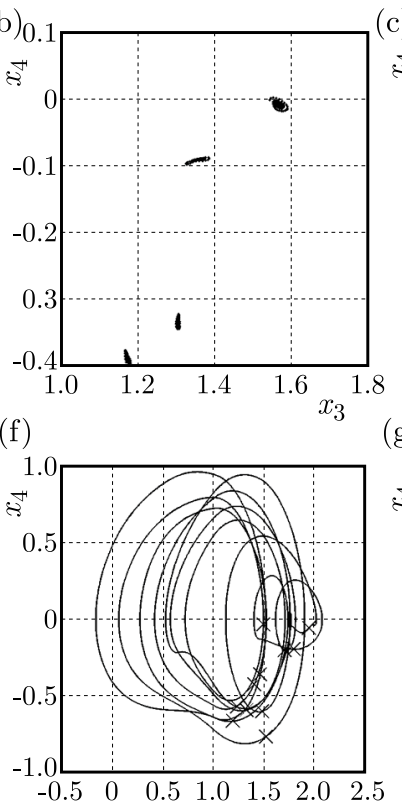

(g)
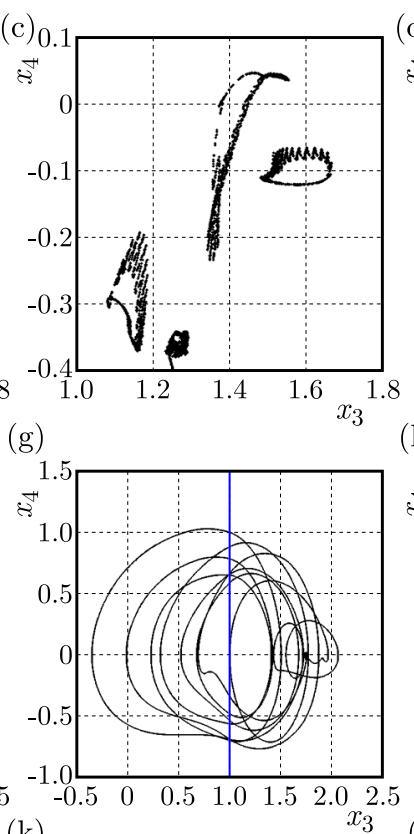

(h)
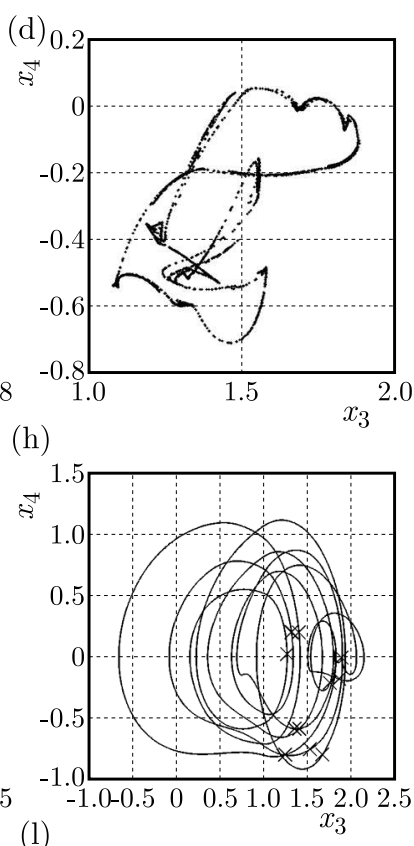

(1)

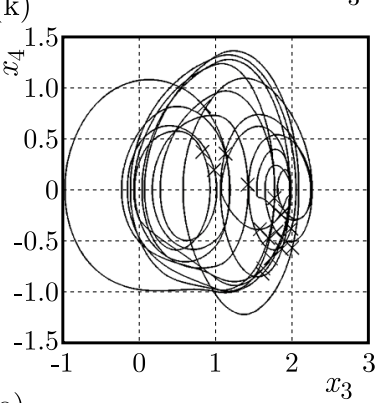

(o)
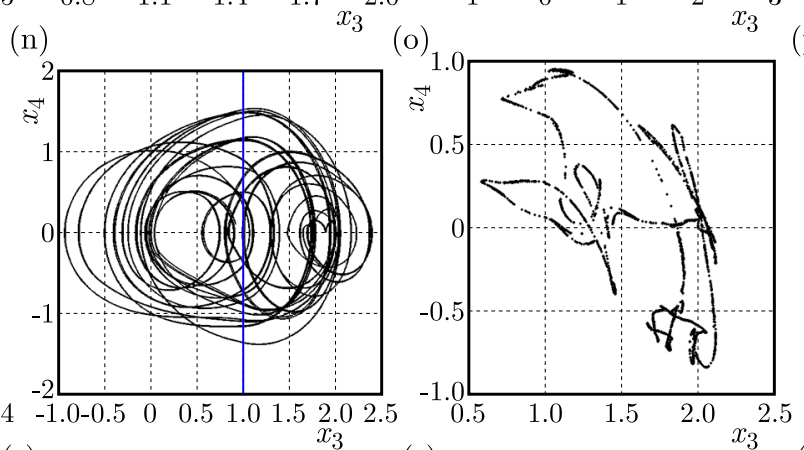

(p)
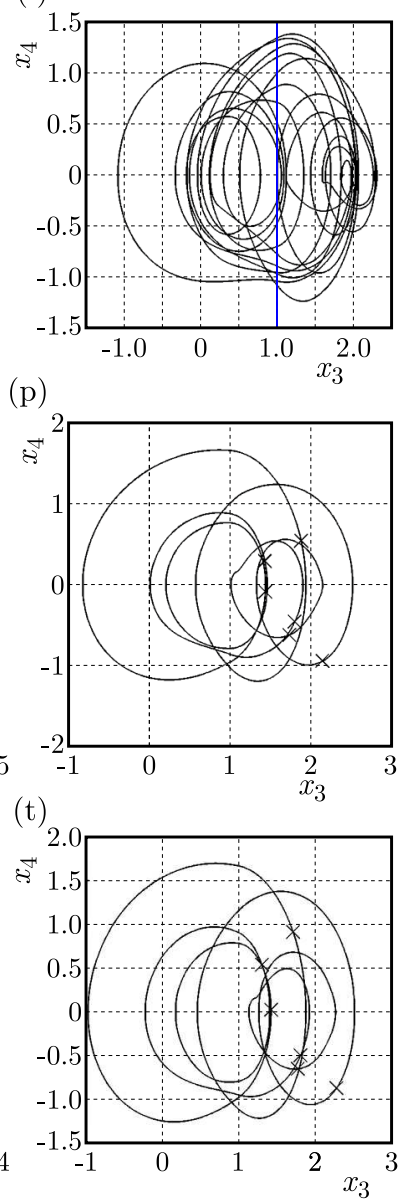

(q)

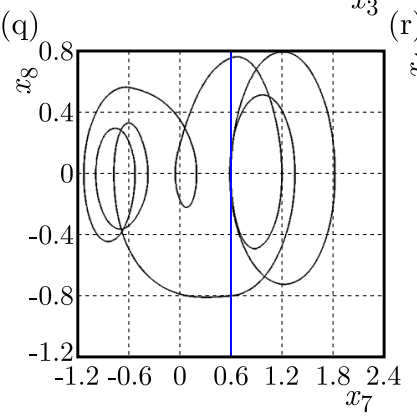

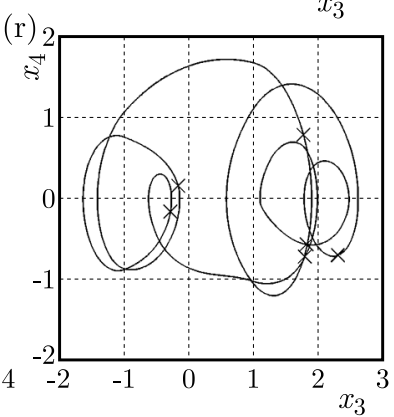

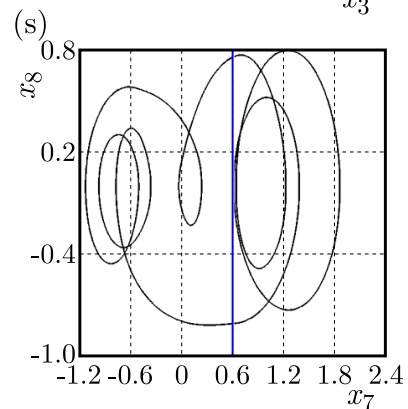

Fig. 4. Phase portraits and diagrams of Poincaré mapping: (a) $\omega=0.99$, (b) $\omega=1.004795$,

(c) $\omega=1.005$, (d) $\omega=1.041$, (e) $\omega=1.05$, (f) $\omega=1.0625$, (g) $\omega=1.06508242$, phase portrait, grazing at the gear surface; (h) $\omega=1.08$, (i) $\omega=1.09205$, phase portrait, grazing at the gear surface;

(j) $\omega=1.1,(\mathrm{k}) \omega=1.13$, (l) $\omega=1.1332$, phase portrait, grazing at the gear surface; (m) $\omega=1.135$,

(n) $\omega=1.1675$, phase portrait, grazing at the gear surface; (o) $\omega=1.18$, (p) $\omega=1.2$, (q) $\omega=1.2087$, phase portrait, grazing at the bearing surface; (r) $\omega=1.21,(\mathrm{~s}) \omega=1.213$, phase portrait, grazing at the bearing surface; (t) $\omega=1.22$. Here, the diagram of Poincaré mapping in the phase portrait is represented by $\times$ and the blue line represents the grazing boundary 
Secondly, the bifurcation and chaos of the system as $\omega \in[0.9,1.3]$ is discussed. The motion of the system is a stable period-1 motion as $\omega<0.9747$. Hopf bifurcation occurs when the frequency increases to $\omega=0.9747$ and it leads the period- 1 motion to quasi-periodic motion. Diagram of Poincaré mapping is illustrated in Fig. 4a as $\omega=0.99$. There is a closed torus in it. The quasi-periodic motion transients to 4 saddle-nodes and they transform to chaos quickly. Diagrams of Poincaré mapping is illustrated in Fig. 4b and Fig. 4c as $\omega=1.004795$ and $\omega=1.005$. The chaos transients into the period-4 motion when the frequency increases to $\omega=1.017$. Saddle-node bifurcation leads the period-4 motion to chaos when the frequency increases to $\omega=1.040798$. The diagram of Poincaré mapping is illustrated in Fig. 4d as $\omega=1.041$. Phase lock occurs when the frequency increases to $\omega=1.0495$ and the diagram of Poincaré mapping is illustrated in Fig. 4e as $\omega=1.05$. The phase lock transients into period-11 motion when the frequency increases to $\omega=1.0623$. The phase portrait and diagram of Poincaré mapping is illustrated in Fig. 4f as $\omega=1.0625$ and the diagram of Poincaré mapping is represented by $\times$. The phase trajectory of the system is tangent to the boundary $x_{3}=1.0$. It implies the grazing occurred in the tooth surface as $\omega=1.06508242$ and its phase portrait is illustrated in Fig. 4g. The phase portrait and diagram of Poincaré mapping is illustrated in Fig. 4h as $\omega=1.08$ after the tooth surface grazing. The number of the periodic motion is not changed but the phase trajectory is changed by the grazing. The phase portraits and diagrams of Poincaré mapping before and after the grazing are shown in Fig. 4f and Fig. 4h. The grazing occurs in the tooth surface again when the frequency increases to $\omega=1.09205$ and it leads the periodic motion to chaos. The phase portrait of the grazing is illustrated in Fig. 4i. The diagram of Poincaré mapping of the chaos is illustrated in Fig. $4 \mathrm{j}$ as $\omega=1.1$ after the tooth surface grazing. The chaotic motion changes to period-17 motion by phase lock when the frequency increases to $\omega=1.1255$. The phase portrait and diagram of Poincaré mapping is illustrated in Fig. $4 \mathrm{k}$ as $\omega=1.13$, and there are 17 discrete points in the diagram of Poincaré mapping. The grazing in the tooth surface leads the periodic motion to chaos when the frequency increases to $\omega=1.1332$, and the phase portrait of the grazing is illustrated in Fig. 4l. The diagram of Poincaré mapping of the chaotic motion is illustrated in Fig. $4 \mathrm{~m}$ as $\omega=1.135$ after the tooth surface grazing. The motion of the system goes to period-23 motion when the frequency increases to $\omega=1.154$. The periodic motion exists in a narrow region only. The grazing occurs in the tooth surface again when the frequency increases to $\omega=1.1657$ and it leads the periodic motion to chaotic motion. The phase portrait of the grazing is illustrated in Fig. 4n. The diagram of Poincaré mapping of the chaotic motion is illustrated in Fig. 40 as $\omega=1.18$ after the tooth surface grazing. The chaos transients to period-6 motion when the frequency increases to $\omega=1.1927$. The phase portrait and diagram of Poincaré mapping is illustrated in Fig. 4p as $\omega=1.2$, and there are 6 discrete points in the diagram of Poincaré mapping. The grazing occurs in the bearing and it leads the phase trajectory of the period- 6 motion change when the frequency increases to $\omega=1.2087$. The phase portrait of the grazing of the bearing is illustrated in Fig. 4q, and the clearance of the bearing is $D_{b}=0.6$. The phase portrait and diagram of Poincaré mapping after bearing grazing is illustrated in Fig. $4 \mathrm{r}$ as $\omega=1.21$. The number of the periodic motion of the system is not changed but the topological shape of the motion is changed by the grazing in the bearing. Grazing occurs in the bearing when the frequency increases to $\omega=1.213$ again, and the phase portrait is illustrated in Fig. $4 \mathrm{~s}$. The period- 6 motion trajectory is changed by the bearing grazing again. The phase portrait and diagram of Poincaré mapping after bearing grazing is illustrated in Fig. 4t as $\omega=1.22$. A conclusion can be drawn that the bearing grazing leads the phase trajectory of the system change but it can not change the number of the system motion. Period-doubling bifurcation occurs when the frequency increases to $\omega=1.25$ and it leads the period-6 motion to period-12 motion. Inverse period-doubling bifurcation occurs when the frequency increases to $\omega=1.2645$ and the period- 12 motion transients into period- 6 motion. 
(a)

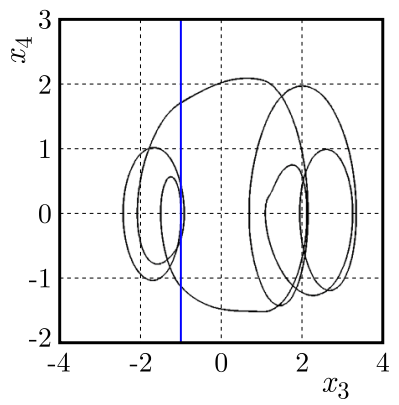

(e)

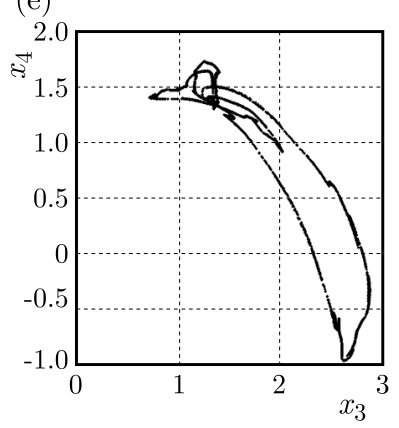

(i)

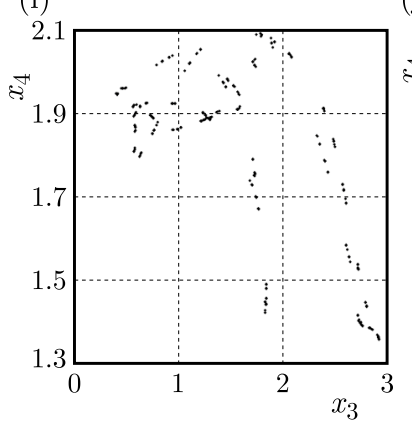

(b)

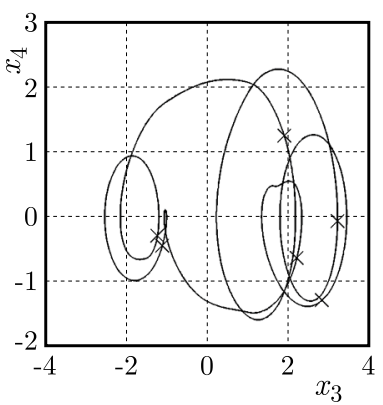

(f)

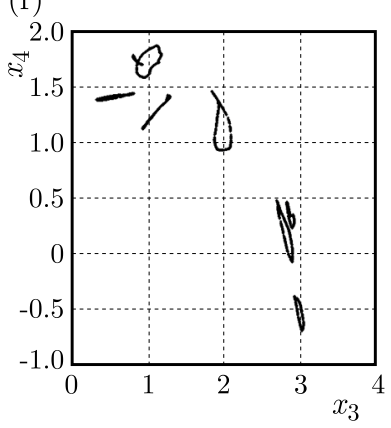

(j)

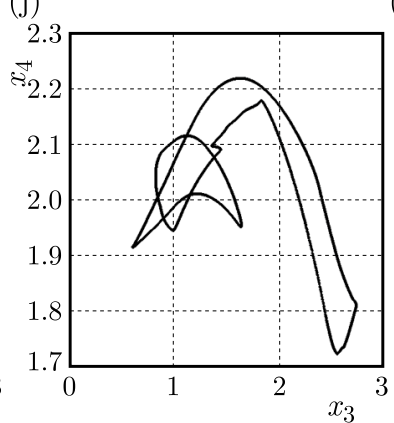

(c)

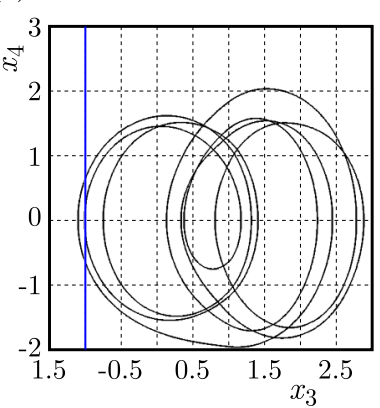

(g)

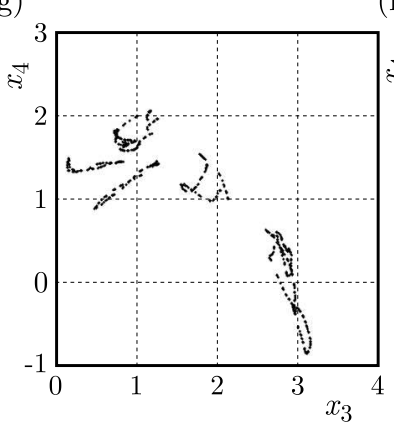

$(\mathrm{k})$

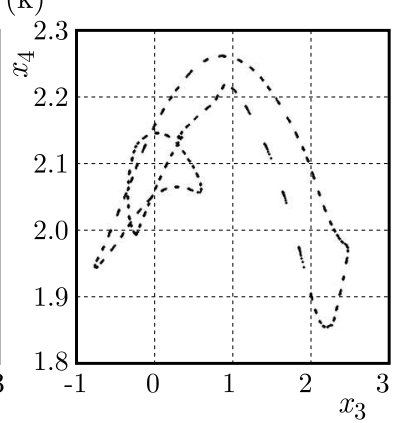

(d)

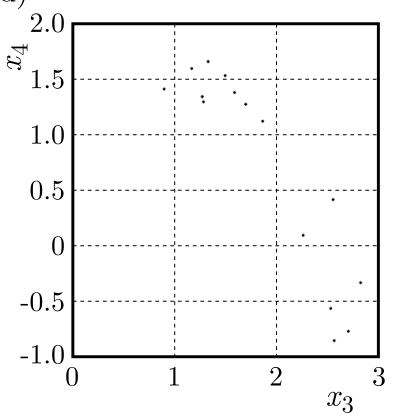

(h)

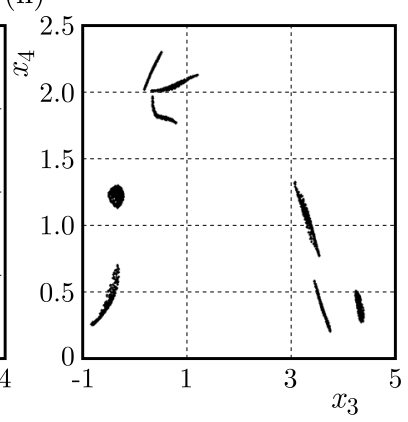

(1)

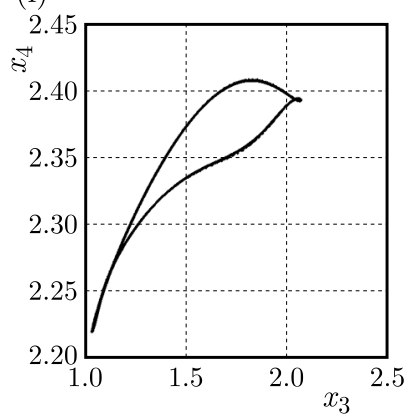

Fig. 5. Phase portraits and diagrams of Poincaré mapping: (a) $\omega=1.3607$, phase portrait, grazing at the tooth back; (b) $\omega=1.38$, (c) $\omega=1.443$, phase portrait, grazing at the tooth back; (d) $\omega=1.45$,

(e) $\omega=1.46$, (f) $\omega=1.575$, (g) $\omega=1.59$, (h) $\omega=1.845$, (i) $\omega=1.85$, (j) $\omega=1.94$, (k) $\omega=1.98$,

(l) $\omega=2.14$. Herein, the diagram of Poincaré mapping in the phase portrait is represented by $\times$ and the blue line represents the grazing boundary

Finally, the bifurcation and chaos of the system as $\omega \in[1.3,2.5]$ is studied. The motion of the system is stable period- 6 as $1.2645<\omega<1.3607$. Grazing occurs at the tooth back and the phase trajectory of the system is changed. The phase portrait of the tooth back grazing is shown in Fig. 5a. The diagram of Poincaré mapping and phase portrait after tooth back grazing is illustrated in Fig. 5b as $\omega=1.38$. The phase trajectory is changed and the number of periodic motion is not changed comparing Fig. 5b to Fig. 4t by the tooth back grazing. Grazing occurs at the tooth back again when the frequency increases to $\omega=1.443$ and the period- 6 motion evolves into period-15 motion. The phase portrait of the tooth back grazing is shown in Fig. 5c as $\omega=1.443$. The diagram of Poincaré mapping after the tooth back grazing is illustrated in Fig. 5d as $\omega=1.45$. The period-15 motion evolves into chaotic motion by saddle-node bifurcation when the frequency increases to $\omega=1.4521$ and the diagram of Poincare mapping is illustrated in Fig. 5e as $\omega=1.46$. Saddle-node bifurcation occurs when the frequency increases to $\omega=1.5412$ and it changes the chaotic motion into period- 8 motion. Hopf bifurcation occurs when the frequency increases to $\omega=1.5697$ and the period- 8 motion degenerates to quasi-periodic motion. The diagram of Poincaré mapping is illustrated in Fig. $5 \mathrm{f}$ as $\omega=1.575$. There are 8 closed tori in the figure. The torus loses its smooth ness and breaks with the increasing 
frequency gradually, and the quasi-periodic motion degenerates to phase lock when the frequency increases to $\omega=1.583$. The diagram of Poincaré mapping is illustrated in Fig. 5g as $\omega=1.59$. The phase lock degenerates to the period- 8 motion when the frequency increases to $\omega=1.6016$. The period- 8 motion goes to chaotic motion by period-doubling bifurcation with an increase in the frequency. The diagram of Poincare mapping is illustrated in Fig. $5 \mathrm{~h}$ as $\omega=1.845$. The phase lock appears again when the frequency increases to $\omega=1.85$. The diagram of Poincaré mapping is illustrated in Fig. 5i as $\omega=1.85$ and it degenerates to period-28 motion when the frequency increases to $\omega=1.889$. Hopf bifurcation occurs when the frequency increases to $\omega=1.921$ and it leads the period-28 motion to a quasi-periodic motion. The diagram of Poincaré mapping is illustrated in Fig. $5 \mathrm{j}$ as $\omega=1.94$. There are 2 closed tori in the figure. The stability of the quasi-periodic motion is lost with an increase in the frequency, and the phase lock appears when the frequency increases to $\omega=1.976$. The diagram of Poincaré mapping is illustrated in Fig. $5 \mathrm{k}$ as $\omega=1.98$. The phase lock evolves into period- 8 motion when the frequency increases to $\omega=2.0799$. Hopf bifurcation occurs when the frequency increases to $\omega=2.12$ and it leads the period-8 motion to quasi-periodic motion again. The diagram of Poincaré mapping is illustrated in Fig. $5 \mathrm{l}$ as $\omega=2.14$. There is a closed torus in the figure. Inverse-Hopf bifurcation occurs when the frequency increases to $\omega=2.1587$ and the quasi-periodic motion degenerates to stable period-1 motion. The period-1 motion exists in a wide region.

The system exhibits complicate dynamical characteristics with an increase in the frequency as above all. Saddle-node bifurcation, Hopf bifurcation, inverse-Hopf bifurcation, period-doubling bifurcation, grazing and so on take place in the process of change of the frequency. Grazing occurs in many areas such as the tooth surface, tooth back and the bearing. The phase trajectory of the system is changed but the number of periodic motion is not changed by the bearing grazing. Not only the phase trajectory but also the number of the periodic motion is changed by grazing at the tooth surface and the tooth back, probably. The gear-rotor-bearing system is a multi-clearance nonlinear system. Clearances exist in the tooth back and the contact surface of the bearing. This may lead to grazing motion in different regions. But the stable simplicity periodic motion can be found in the gear-rotor-bearing system according to the above analysis when the frequency is taken to a reasonable value. The motion of the system remains stable period-1 motion when the frequency is in the range of $\omega \in[0.602,0.9747]$ and $\omega>2.1587$.

\subsection{Effect of the time-varying stiffness}

The bifurcation diagram of the relative displacement via the amplitude of the time-varying stiffness $k$ is illustrated in Fig. 6 when the values of dimensionless parameters are taken from Table 3 and $\omega=1.05$. The motion of the system is a long-periodic motion when the amplitude of the time-varying stiffness is $k<0.1032$ as shown in Fig. 6 . The long-periodic motion evolves into quasi-periodic motion by Hopf bifurcation when the amplitude of the time-varying stiffness is $k=0.1032$. The quasi-periodic motion degenerates to long-periodic motion again when the amplitude of the time-varying stiffness increases to $k=0.1173$. The long-periodic motion degenerates to quasi-periodic motion by Hopf bifurcation again when the amplitude of the time-varying stiffness increases to $k=0.1332$. Two tori in these two quasi-periodic motions are irregular and the irregularity leads the quasi-periodic motion unstable. The motion of the system shocks between the long-periodic motion and the quasi-periodic motion when the amplitude of the time-varying stiffness $k$ exists in the range of $[0.1332,0.2556]$. The motion of the system moves to period-10 motion by inverse-Hopf bifurcation as $k=0.2556$. The phase portrait and diagram of Poincaré mapping are illustrated in Fig. 7a as $k=0.26$. There are 10 discrete points. which implies that the motion of the system is stable period-10 motion. Grazing occurs in two bearings and it makes the phase trajectory of the system change. Phase portraits of the grazing are illustrated in Fig. 7b and Fig. 7c. The phase portrait and diagram of Poincaré mapping after 
grazing is illustrated in Fig. $7 \mathrm{~d}$ as $k=0.28$. The periodic motion trajectory is changed slightly but the motion property is not changed by the grazing comparing Fig. 7a to Fig. 7d. It is said that the grazing of the bearing makes the motion trajectory change only. Grazing occurs in the reverse direction of two bearings simultaneously. It implies that grazing is caused by the lean of the shaft in the meshing process, probably. The motion of the system is possibly changed by the assemblement precision of the gear system and the lean of the shaft in the movement. The high assemblement precision of the gear system is used to obtain high movement stability in the meshing in practical engineering.

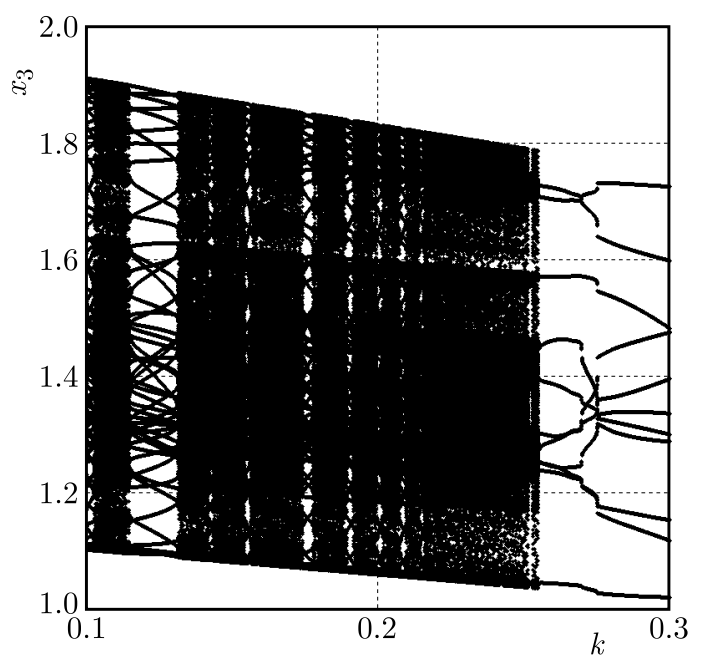

Fig. 6. Bifurcation diagram of system via the amplitude of the time-varying stiffness

(a)

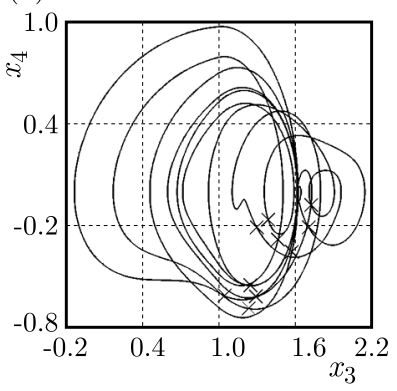

(b)

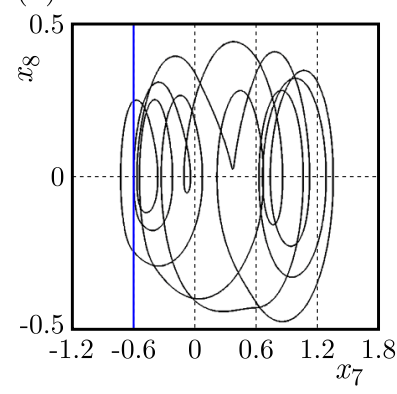

(c)

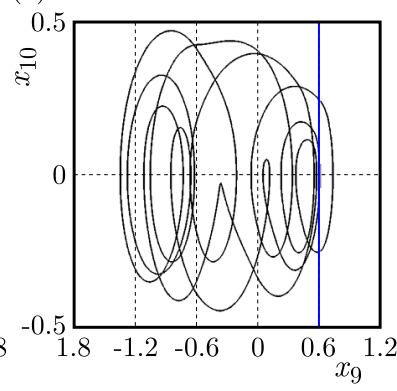

(d)

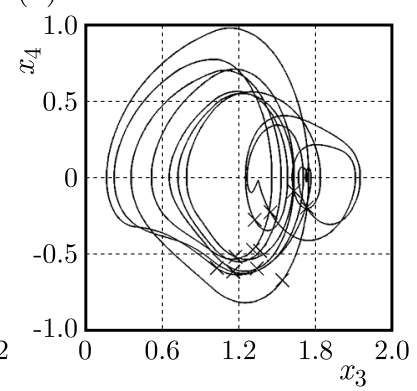

Fig. 7. Phase portraits and diagrams of Poincaré mapping: (a) $k=0.26$, (b) $k=0.272$, phase portrait, grazing in the bearing; (c) $k=0.272$, phase portrait, grazing in the bearing; (d) $k=0.26$. Here, the diagram of Poincaré mapping is represented by $\times$ and the blue line represents the grazing boundary

The motion of the system exhibits nonlinear characteristics when the stiffness is small but a stable periodic motion can be obtained as the stiffness is large when other parameters are the same. The quench is used to improve the meshing stiffness. High meshing stiffness is used to obtain stable periodic motion in practical engineering.

\subsection{Effect of the bearing clearance}

The bearing clearance $D_{b}$ has an essential influence on the dynamical behavior of the gear-rotor-bearing system. The bifurcation diagrams of the relative displacement via the bearing clearance $D_{b}$ are illustrated in Fig. 8 when the values of dimensionless parameters are taken from Table 3 and $\omega=1.05$. Figure $8 \mathrm{a}$ is the bifurcation diagram of the system for the increasing bearing clearance and Fig. $8 \mathrm{~b}$ is the bifurcation diagram of the system for the decreasing bearing clearance. 
(a)

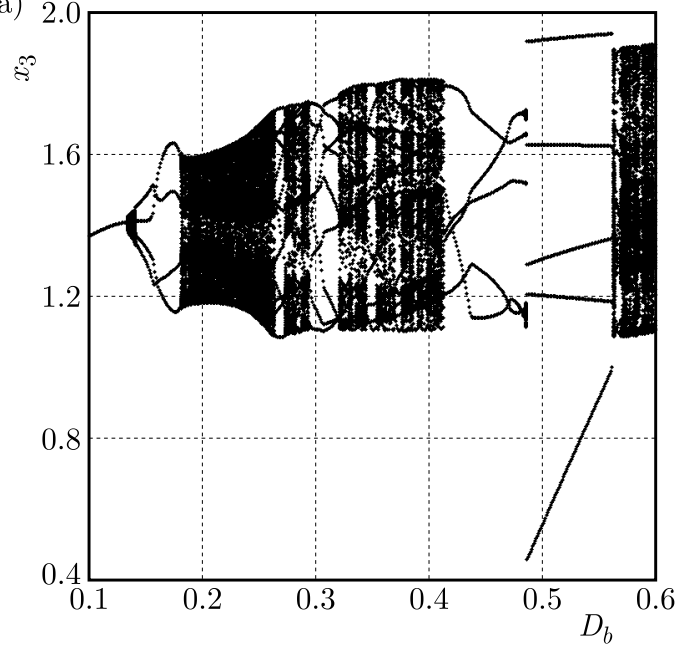

(b)

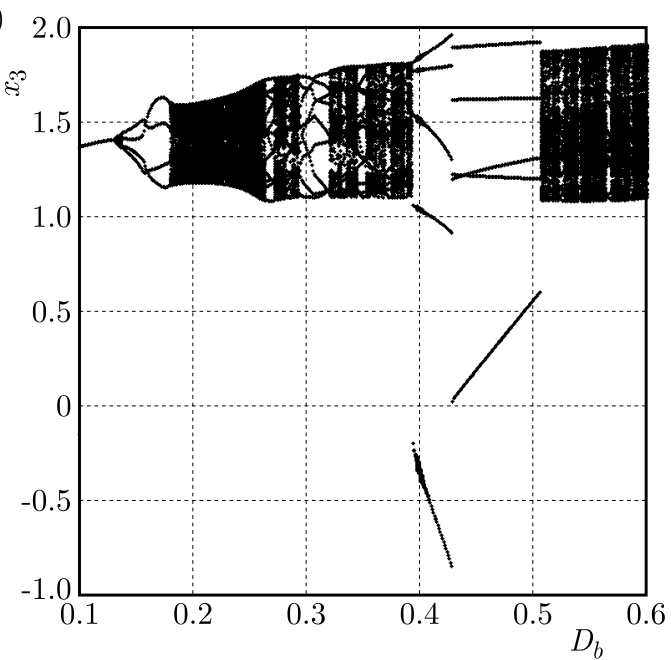

Fig. 8. Bifurcation diagrams of system via the bearing clearance, $D_{b}=[0.1,0.6]$ : (a) with the increasing of the bearing clearance; (b) with the decreasing of the bearing clearance

The motion of the system is stable period- 1 when the bearing clearance is lower than 0.132 as shown in Fig. 8a. Hopf bifurcation leads the period-1 motion to quasi-periodic motion when the bearing clearance increases to $D_{b}=0.132$. It is a closed limit torus in the diagram of Poincaré mapping. The smoothing of the limit torus loses gradually, the distortion and breakage occur. The motion of the system evolves into period-4 motion when the bearing clearance increases. The period-4 motion evolves into quasi-periodic motion by Hopf bifurcation when the bearing clearance increases to $D_{b}=0.1815$. It is an irregular closed limit torus. The irregularity of the closed limit torus directs the motion of the system unstable. The motion of the system shocks between the periodic motion and quasi-periodic motion in the region of $D_{b} \in[0.23,0.295]$. The quasi-periodic motion evolves into period-13 motion when the bearing clearance increases to $D_{b}=0.261$. The period-13 motion changes to quasi-periodic motion when the bearing clearance increases to $D_{b}=0.272$. The quasi-periodic motion evolves into period-9 motion when the bearing clearance increases to $D_{b}=0.294$. The motion of the system shocks between the periodic motion and quasi-periodic motion in the region of $D_{b} \in[0.321,0.413]$. The motion of the system evolves into period-5 motion by the phase lock when the bearing clearance is greater than 0.413 . The periodic motion evolves into quasi-periodic motion when the bearing clearance increases to $D_{b}=0.4798$ but it exists in a narrow region. It degenerates to period-5 motion again when the bearing clearance increases to $D_{b}=0.484$. The phase lock occurs again when the bearing clearance increases to $D_{b}=0.5618$.

There is a coexistence of attractors in the region of $D_{b} \in[0.3935,0.5612]$ according to Fig. 8. The diagrams of Poincaré mapping are shown in Fig. 9 for $D_{b}=0.4,0.44,0.5,0.52$, respectively. Different colors represent different attractors of the coexistence. The red one represents the attractor with the increasing bearing clearance and the black one represents the attractor with the decreasing bearing clearance. The coexistence of attractors for periodic motion and phase lock, periodic motion and quasi-periodic motion, periodic motion and periodic motion and so on in the region is discovered.

The bearing clearance affects the nonlinear dynamic behavior of the system. The motion of the system is stable when the value of the bearing clearance is small, and it is complicated when the clearance is big according to the analysis above. The motion of the system is stable period-1 motion when the bearing clearance is less than 0.12 . The motion of the system is stable periodic motion but there is coexistence of attractors when the bearing clearance is in the region of $D_{b} \in[0.41,0.5]$. This coexistence means strong nonlinear characteristics because the motion 
(a)

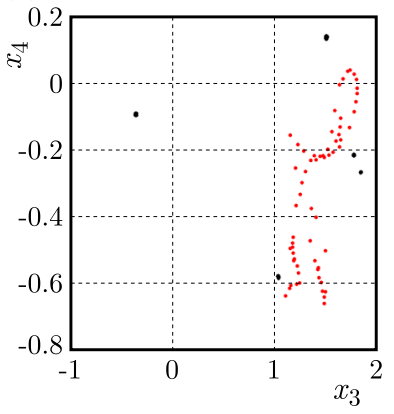

(b)

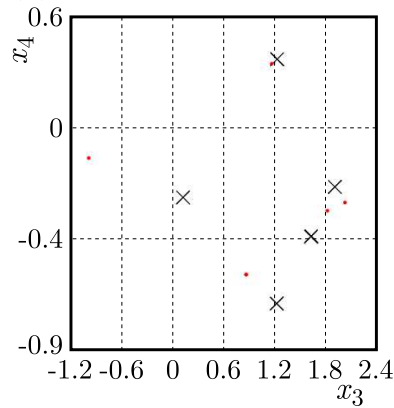

(c)

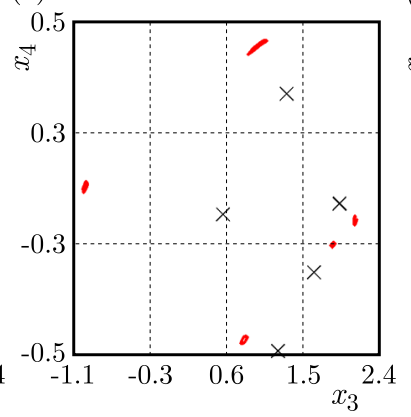

(d)

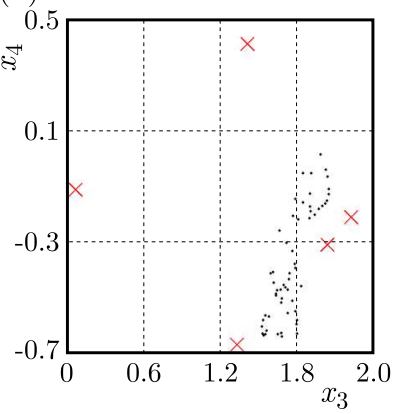

Fig. 9. Diagrams of Poincaré mapping: (a) $D_{b}=0.4$, (b) $D_{b}=0.44$, (c) $D_{b}=0.5$, (d) $D_{b}=0.52$. Here, red color represents the diagram of Poincaré mapping with the increasing bearing clearance and black color represents the one with the decreasing bearing clearance

form will change with a change in the initial value. A small value of the bearing clearance should be selected to obtain stable motion of the gear system. The bearing clearance should be selected as $40 \%-50 \%$ of the backlash if the small bearing clearance can not be obtain by manufacturing precision. Noise and vibration increase when the degree of wear reaches a certain degree in engineering practice. Big bearing clearance causes strong impact and vibration because of wear of rolling elements. So a small bearing clearance should be selected and the worn bearing should be replaced to decrease the impact and vibration and increase the stability of the system.

\subsection{Effect of the comprehensive transmission error and backlash}

The bifurcation diagrams of the relative displacement versus the amplitude of the comprehensive transmission error $\varepsilon$ are illustrated in Fig. 10a. The values of dimensionless parameters are taken from Table 3 and $\omega=1.05$. The motion of the system is stable period- 5 when the amplitude of the comprehensive transmission error is less than 0.7316 as shown in Fig. 10a. There are 5 discrete points in the diagram of Poincaré mapping. The periodic motion evolves

(a)

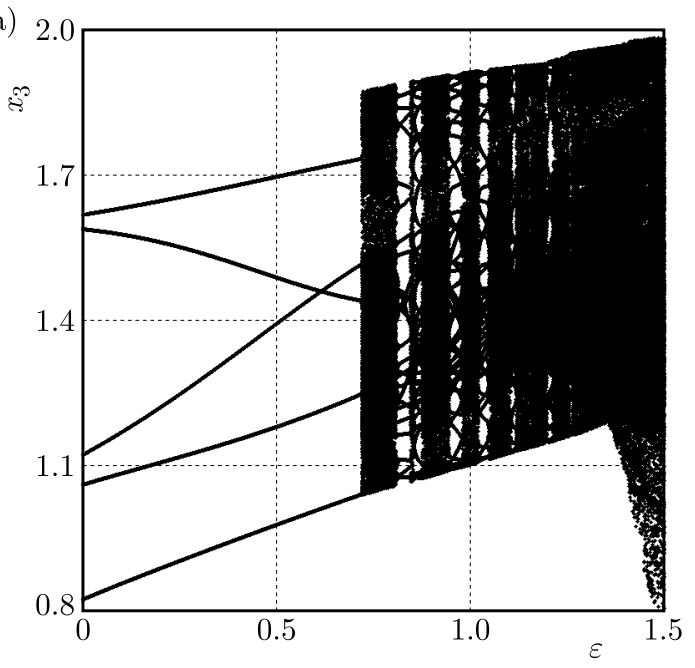

(b)

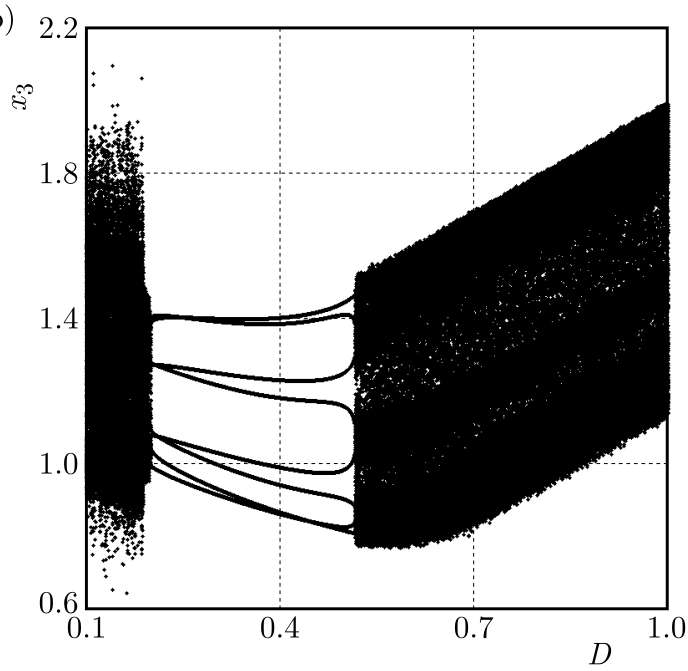

Fig. 10. Bifurcation diagrams of the system: (a) vs. the transmission error, (b) vs. backlash

into chaotic motion when the amplitude of the comprehensive transmission error increases to $\varepsilon=0.7316$. The motion of the system shocks between the long-periodic motion and chaotic motion with the increasing amplitude of the comprehensive transmission error. The region of the long-periodic motion becomes narrow and the region of the chaotic motion becomes wide 
with a gradual increase in the amplitude of the comprehensive transmission error. The motion of the system is chaotic in this region. The morphology of the chaotic motion change when the amplitude of the comprehensive transmission error increases to $\varepsilon=1.4108$.

The motion of the system becomes instable with an increase in the amplitude of error (comprehensive transmission error of the system). Chaotic motion, shock of chaotic motion and periodic motion occur with an increase in the amplitude of the error, and the instability of the system becomes worse. The motion of the system preserves stable when the value of the amplitude of the error is low. So the manufacturing and assemblement precision of the gear should be improved to obtain stability of the system.

The bifurcation diagram of the relative displacement versus the backlash $D$ is illustrated in Fig. 10b. The motion of the system is period- 8 when the backlash is in the region of $D \in[0.2004,0.5154]$. There are 8 discrete points. The motion of the system is chaotic when the backlash is less than 0.2004 and greater than 0.5154. Backlash is an important factor to lead the motion of the system to chaos. Chaos is caused by rattling when the backlash is small, and it is caused by impact when the backlash is big. So a reasonable backlash must be selected to improve the stability of the gear system.

\section{Conclusion}

A nonlinear dynamic model of a gear-rotor-bearing system is established when the time-varying stiffness, tooth surface friction, backlash, bearing clearance and the comprehensive transmission error are considered. The effects of frequency, backlash, bearing clearance, comprehensive transmission error and stiffness on dynamic characteristics of the system are analyzed. Some conclusions can be drawn according to the analysis.

Firstly, there are some complicated nonlinear phenomena such as grazing bifurcation, quasi-periodic bifurcation, chaos and coexistence of attractors in the system. Stable periodic motion can be obtained by low backlash, bearing clearance, comprehensive transmission error, high manufacturing and assemblement precision of the gear and mesh stiffness.

Secondly, the phase trajectory and the number of periodic motion can be changed by the grazing at the tooth surface and the tooth back. Different kinds of grazing motions may be produced by clearances existing in the gear back and the contact surface of the bearing. But a stable periodic motion can be obtained when the frequency is taken to a reasonable value.

Finally, the phase trajectory of the system should be changed but the number of the periodic motion can not be changed by the bearing grazing. A small value of the bearing clearance should be selected to obtain stable motion of the gear system. Sometimes, grazing occurs in the reverse direction of two bearings simultaneously. This means that the grazing is caused by the lean of the shaft in the meshing process. The motion of the system is changed by the assemblement precision of the gear system and the lean of the shaft in the movement. High assemblement precision of the gear system should be secured to obtain high movement stability of the meshing in the engineering practice.

Acknowledgments

This work was financially supported by the National Natural Science Foundation of China (grant No. 51365025), by Natural Science Key Foundation of Tianjin, China (grant No. 16JCZDJC38500) and by the Natural Science Foundation of Tianjin, China (grant No. 17JCQNJC04300).

\section{References}

1. Chang-Jian C.J., 2010a, Non-linear dynamic analysis of a HSFD mounted gear-bearing system, Nonlinear Dynamics, 62, 333-347 
2. Chang-Jian C.J., 2010b, Strong nonlinearity analysis for gear-bearing system under nonlinear suspension - bifurcation and chaos, Nonlinear Analysis: Real World Applications, 11, 1760-1774

3. Chang-Jian C.J., Chang S.M., 2012a, Bifurcation and chaos analysis of the porous squeeze film damper mounted gear-bearing system, Computers and Mathematics with Applications, 64, 5, 798-812

4. Chang-Jian C.J., Chang S.M., 2012b, Chaotic responses on gear pair system equipped with journal bearings under turbulent flow, Applied Mathematical Modelling, 36, 6, 2600-2613

5. Chen S.Y., Tang J.Y., Luo C.W., 2011, Nonlinear dynamic characteristics of geared rotor bearing systems with dynamic backlash and friction, Mechanism and Machine Theory, 46, 4, 466-478

6. Choi S.T., MAU S.Y., 2001, Dynamic analysis of geared rotor-bearing systems by the transfer matrix method, Journal of Mechanical Design, 123, 562-579

7. Cui Y.H., LiU Z.S., WANG Y.L., 2012, Nonlinear dynamic of a geared rotor system with nonlinear oil film force and nonlinear mesh force, Journal of Vibration and Acoustics, Transactions of the ASME, 134, 4, 1313-1320

8. Kahraman A., Singh R., 1990, Non-linear dynamics of a spur gear pair, Journal of Sound and Vibration, 142, 1, 49-75

9. Kahraman A., Singh R., 1991, Non-linear dynamics of a geared rotor-bearing system with multiple clearances, Journal of Sound and Vibration, 144, 3, 135-156

10. Ranghothama A., NARAyAnAn S., 1999, Bifurcation and chaos in geared rotor bearing system by incremental harmonic balance method, Journal of Sound and Vibration, 226, 3, 469-492

11. Theodossiades S., Natsiavas S., 2001, On geared rotor dynamic systems with oil journal bearings, Journal of Sound and Vibration, 243, 4, 721-745

12. XiAng L., JiA Y., Hu A., 2016, Bifurcation and chaos analysis for multi-freedom gear-bearing system with time-varying stiffness, Applied Mathematical Modelling, 40, 10506-10520 